\title{
Muon Track fast Tag : A muon trigger upgrade for CMS at the HL-LHC
}

\author{
Oliver Pooth ${ }^{* \dagger}$ \\ RWTH Aachen \\ E-mail: pooth@physik.rwth-aachen.de
}

Triggering on high $p_{t}$ muons at the projected high luminosity LHC (HL-LHC) with an expected instantaneous luminosity of $10^{35} /\left(\mathrm{cm}^{2} \cdot \mathrm{s}\right)$ will be one major challenge for the CMS experiment from 2022 on. With the Muon Track fast Tag (MTT) a new detector subsystem is proposed that can help to keep the Level-1 trigger rate low enough without increasing the $p_{t}$ thresholds for single muons. The trigger concept must be extended by combining the information of the inner tracking system with a fast muon tag which is also able to reduce the problem with ambiguities of simultaneously traversing muons leading to so-called ghost hits.

Tiles of fast plastic scintillator material read out by silicon photomultipliers (SiPMs) are under investigation to provide the additional detector layer in the very limited space available between the CMS solenoid and the first muon stations.

36th International Conference on High Energy Physics

4-11 July 2012

Melbourne, Australia

* Speaker.

On behalf of the CMS collaboration. 


\section{Motivation for a Muon Track fast Tag (MTT) at CMS}

From the year 2022 the CMS experiment at the LHC is expected to operate at the high luminosity LHC (HL-LHC). Up to $1000 \mathrm{fb}^{-1}$ of data will be collected per year with an instantaneous luminosity that will be increased by a factor of ten compared to the running LHC with a design luminosity of $10^{34} /\left(\mathrm{cm}^{2} \cdot \mathrm{s}\right)$. New physics scenarios and extended limits which are expected and hoped for in the coming years are displayed in Figure 1 where the integrated luminosity is shown versus time. Various scenarios are under discussion to increase the amount of data to search for events with extremely small cross sections, e.g. increasing beam energy, higher bunch crossing frequency and higher number of protons per bunch.

To trigger on high $p_{t}$ muons at $10^{35} /\left(\mathrm{cm}^{2} \cdot \mathrm{s}\right)$ while keeping the Level-1 trigger rate low the trigger concept needs to be extended. With the MTT (see Figure 1, right), a fast 2D trigger system located between the CMS solenoid and the inner muon stations is proposed in [2] to cope with high muon rates without increasing the $p_{t}$ thresholds. Just increasing the threshold would be an inefficient way as shown in Figure 2, mainly due to poorly reconstructed muon $p_{t}$ on Level-1.

By combining the information from various subsystems the muon momentum resolution can be improved in the Level-1 trigger system. The inner silicon based tracking system provides an excellent $p_{t}$ measurement and with the MTT it is possible to define regions of interest for the tracker in case of high $p_{t}$ muons. In addition the MTT can resolve possible muon ambiguities at very high luminosities (ghost rejection).
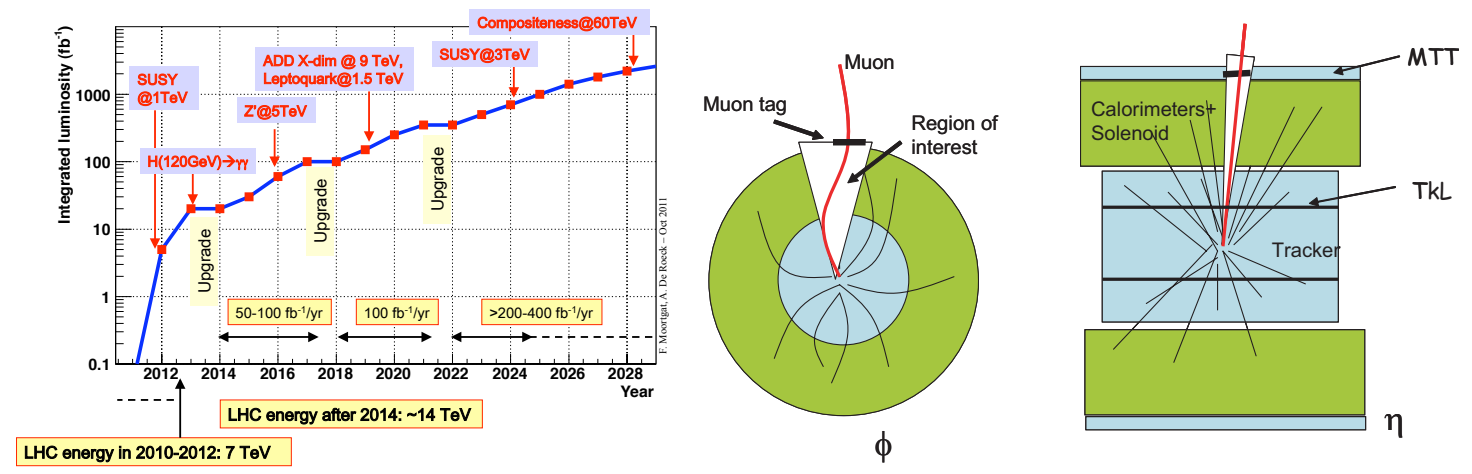

Figure 1: Left: The integrated luminosity versus time for the periods before, between and after the three long shutdowns to come [1]. Right: The Muon Track fast Tag concept [2].

\section{Constraints and consequences for detector modules}

Several constraints need to be respected before the technology choice can be made and prototype detector modules can be qualified to the specifications necessary for the operation at HL-LHC conditions. The very limited space in the region between the CMS solenoid and the inner muon stations requires thin detector modules. To provide a fast trigger the detector signals must be fast to allow for a reliable bunch crossing identification. The additional detector layer must be operated in a high magnetic field. As a consequence of these constraints first prototype modules were built based on fast plastic scintillator tiles read out by silicon photomultipliers (SiPMs), arrays of avalanche photo diodes operated in Geiger mode. 


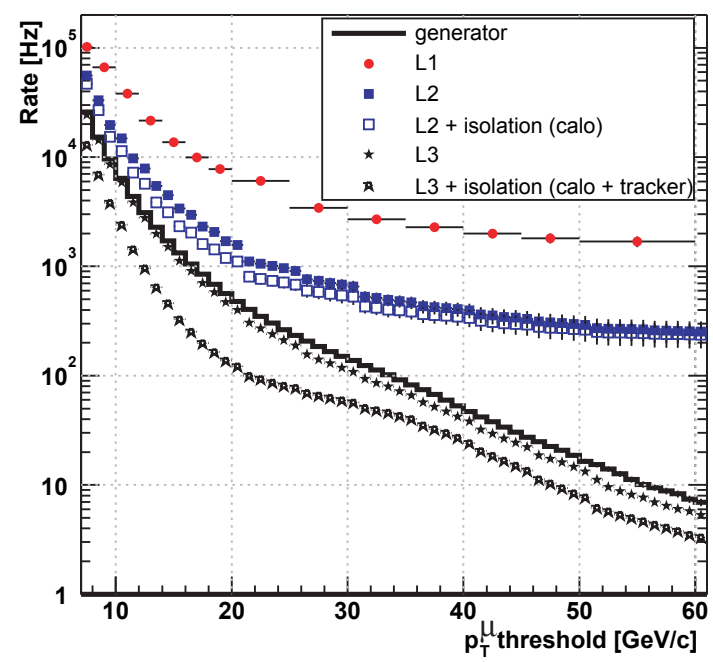

Figure 2: Rates for different trigger levels as a function of the muon $p_{t}$ threshold [3].

\subsection{Prototype modules}

First prototypes (see Figure 3) are based on diamond tool finished BC-404 (Saint Gobain, [4]) scintillator tiles. Wave length shifting fibres (BCF-72, [4]) on both sides of the scintillator tile with $1 \mathrm{~mm}$ in diameter are used to guide the scintillation light to two SiPMs (100 pixels on $1 \times 1 \mathrm{~mm}^{2}$ ) [5]. A characteristic pulse height spectrum read out by SiPMs is shown in Figure 3 (right).
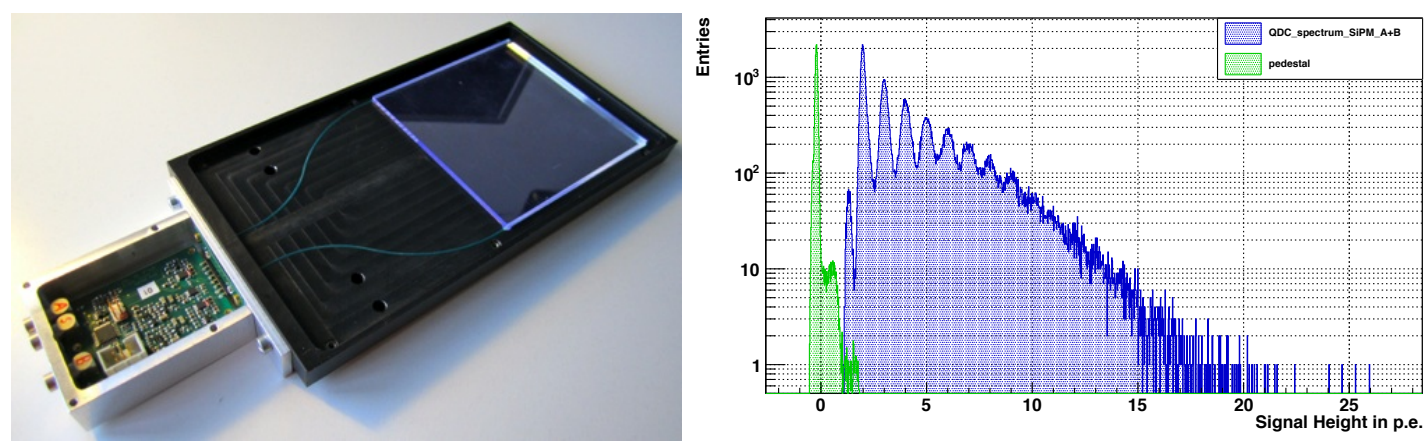

Figure 3: Left: Photo of a prototype module. Right: Characteristic pulse height spectrum recorded with a charge-to-digital converter (QDC) and electrons emitted on the detector module by a ${ }^{90} \mathrm{Sr}$ source. Individual peaks belong to the corresponding number of pixels fired and are called photon equivalent peaks (p.e.).

Figure 4 shows the amplifier and controller [6] developed at the 3. Physikalisches Institut B, RWTH Aachen university, that controls and amplifies the signals of the two SiPMs on the front end board. The signal purity as a function of the signal discriminator threshold is shown in Figure 5. The detection efficiency for cosmic muons and this particular module was determined to be $95.9_{-0.3}^{+0.2} \%$. Alternative prototypes with large SiPMs $\left(900\right.$ pixels on $\left.3 \times 3 \mathrm{~mm}^{2}\right)$ and without wavelength shifting fibres have shown efficiencies above $98 \%$ for cosmic muons [7].

Silicon photomultipliers suffer from large temperature dependencies (dark counting rate, leakage current, etc.). Active temperature compensation is provided by a microcontroller on the front 

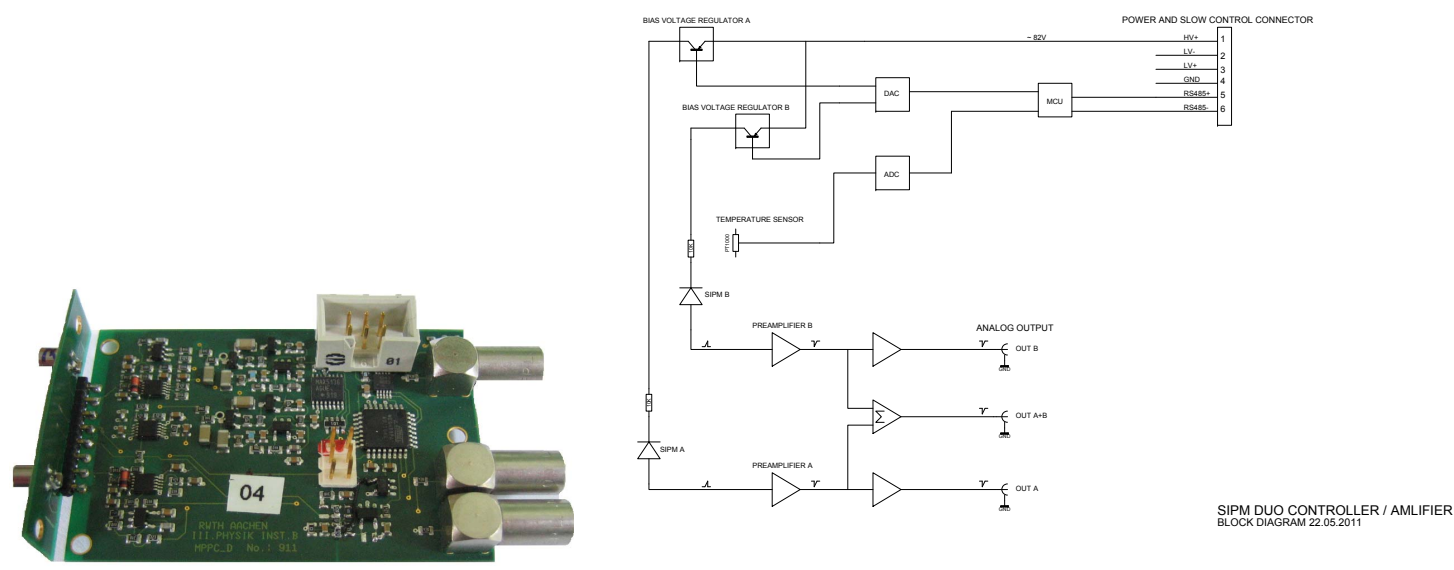

Figure 4: Left: Photo of the SiPM Duo Controller / Amplifier. Right: Block schematics of the SiPM Duo Controller / Amplifier [6].

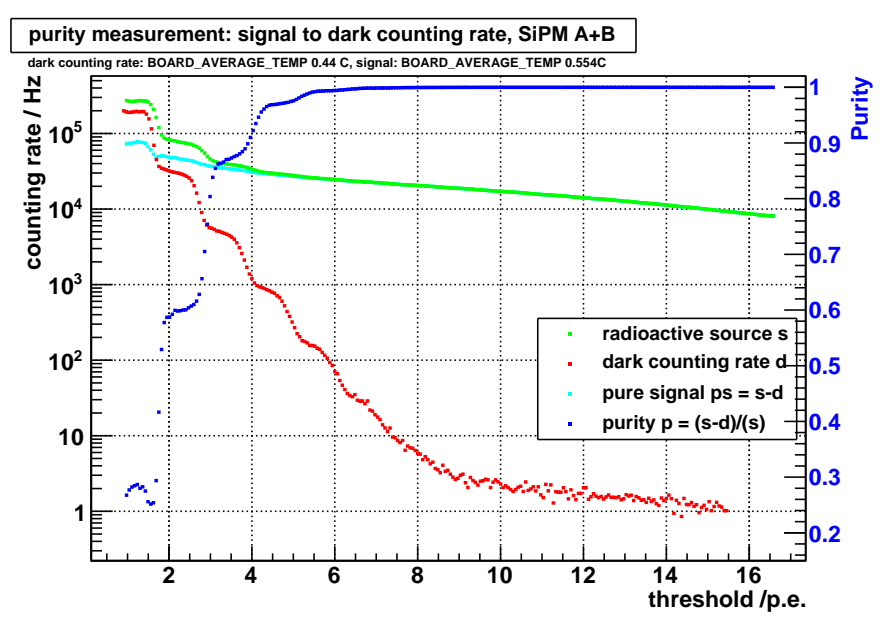

Figure 5: Various counting rates are displayed and used to determine the signal purity vs. the signal threshold. Requiring a signal height corresponding to 5 p.e. a signal purity of $>99 \%$ is achieved (right scale).

end and the effect is shown in Figure 6. With the temperature correction coefficient of $57 \mathrm{mV} / \mathrm{K}$ characteristic peaks in the SiPM spectra stay in position. No temperature dependency of the peak positions is visible anymore.

\subsection{Alternative gain stabilization}

Instead of adjusting the bias voltage according to the measured ambient temperature the characteristic SiPM spectrum itself can be used to stabilize the gain of the device. With a PID algorithm acting on data taken by a multilevel discriminator it is possible to stabilize the position of the 1 p.e. peak independent of the externally measured temperature. Figure 7 shows the bias voltage and the ambient temperature. The bias voltage was determined by the PID algorithm and applied to the SiPMs. 

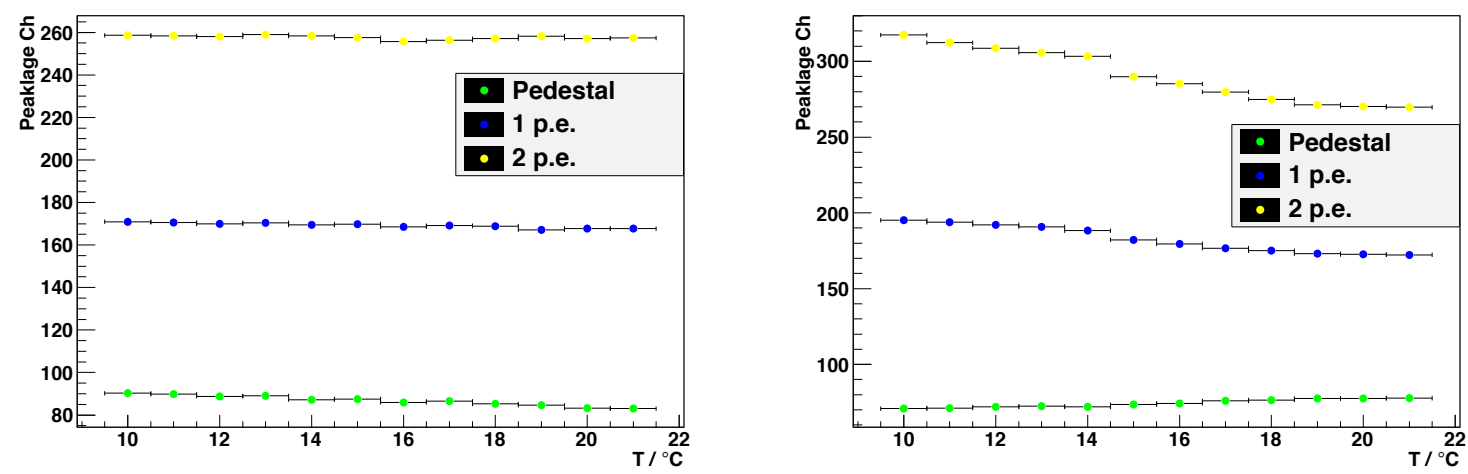

Figure 6: Characteristic SiPM peak positions (and pedestal) versus ambient temperature with temperature compensation switched on (left) and temperature compensation switched off (right) [8].

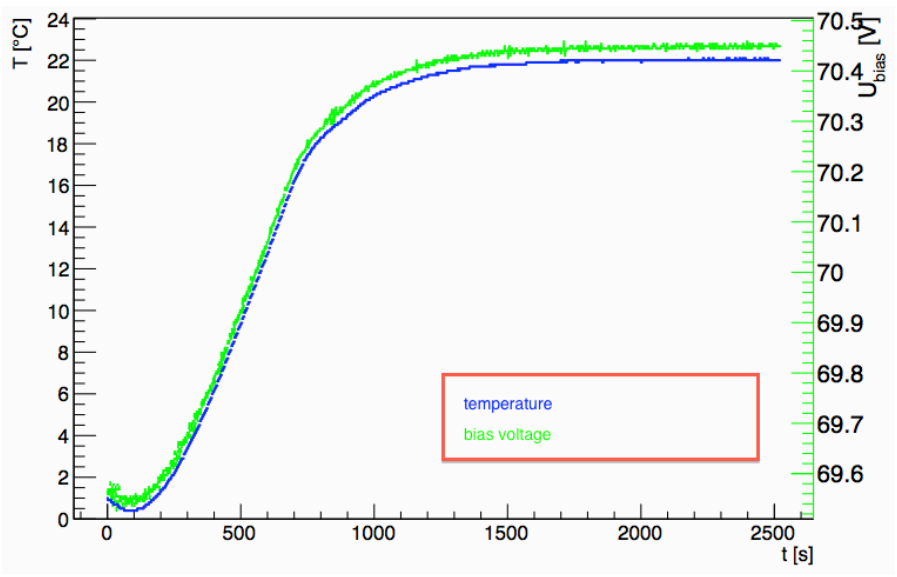

Figure 7: The plot shows the applied bias voltage needed to keep the 1 p.e. peak in stable position (right scale). The second curve shows the ambient temperature (left scale) as control variable [9].

\section{Outlook}

A virtual MTT detector layer is now being integrated in the CMS software framework to study the optimal detector granularity for HL-LHC luminosities and ghost rejection capabilities. In addition it is essential to understand the slow heavy ionizing particles before finalizing the design. Other prototypes without wave length shifting fibres and the behavior of alternative fibre routing are studied to find the optimal single module design before taking the step towards a detector system. To step up from prototype modules to larger substructures, system aspects need to be studied, e.g. power consumption, cooling, read out and triggering. In parallel, SiPM characteristics must be understood in greater detail. Photon detection efficiencies, cross talk between neighbouring SiPM cells and afterpulsing in single cells must be studied in dependence of $U_{\text {bias }}$ and are under investigation at the 3. Physikalisches Institut A, RWTH Aachen university. Finally, SiPMs with realistic response characteristics need to be implemented in GEANT 4 to study the MTT system in greater detail. For the coming years all simulations must be complemented with test setups and finally beam tests. 


\section{References}

[1] A. de Roeck, F. Moortgat, CMS internal communication.

[2] A. Montanari et al., Muon Trigger Upgrade at SLHC: Muon Track fast Tag, internal note CMS IN $-2007 / 058$.

[3] The CMS collaboration, Technical Proposal for the Upgrade of the CMS detector through 2020, CERN-LHCC-2011-006.

[4] BC-404 data sheet on http://www.detectors.saint-gobain.com/.

[5] Multi-Pixel Photon Counter S10362-11-100U, Hamamatsu solid state division, August 2009.

[6] F. Beissel, SiPM Duo Controller / Amplifier, V1.10, 20.07.2011, internal documentation available from the author.

[7] M. Quittnat, A Test Bench for the Optimization of Scintillation Detectors read out by Silicon Photomultipliers for the MTT System at CMS, Master thesis RWTH Aachen, September 2012.

[8] M. Emde, Inbetriebnahme und Untersuchung eines per Silizium-Photomultiplier ausgelesenen Szintillationsdetektors zur Parameteroptimierung, Bachelor thesis RWTH Aachen, September 2011.

[9] L.S. Weinstock, Regulierung der Versorgungsspannung von Silizium-Photomultipliern anhand ihrer charakteristischen Spektren, Bachelor thesis RWTH Aachen, September 2012. 